\section{PROTECTIVE VISORS IN SEVEN COLOURS}

QED offer a range of extremely cost-effective protective visors, Comfort Relax and Comfort Light. Suitable for use in all aspects of dental treatment they are extremely comfortable to wear and protect the wearer's face from all the 'splatter ' $n$ ' spray' associated with most dental procedures. Lightweight and hygienic they can be worn in association with conventional corrective glasses.

Available in a choice of seven colours, including white and clear, they are available in two types. Comfort Relax has metal clips and can be used in conjunction with all the types of foil currently available on the market. Comfort Light has the lightest frame on the market and features special plastic holders rather than metal clips. Whichever style the wearer prefers they are supplied with four Comfort Foils which deliver the highest translucency parameter of 99.97\%, effectively providing crystal clear clarity. Both Comfort Relax and Comfort Light are available for just $£ 15.00$ plus VAT, while refill packs of four Comfort Foils cost just £7.00 plus VAT.

For further information telephone Quality Endodontic Distributors Ltd on 01733404 999, emailsales@qedendo. co.uk, visit www.qedendo. co.uk or contact your local QED salesperson.

\section{IT'S SUCH A GOOD VIBRATION}

Close to 100 dental practices in the UK are now using Vibraject, the small vibrating device which attaches to a dental syringe and removes or reduces the pain of an injection, is currently catching on in the dental profession.

As part of the campaign, a new website - www. vibraject.co.uk - was launched in February. It includes patient and dentist testimonials as well as a list of dental practices across the country which have Vibraject. Dental professionals who invest in Vibraject are given stickers so they can proclaim and promote their commitment to painfree dentistry with a 'Vibraject here' sign.

It's estimated that $25 \%$ of adults in the UK delay seeking help for a painful dental condition due to dental anxiety. For many, the anxiety is caused by needle phobia. When Vibraject is used to give a local anaesthetic, many patients don't even know they have had an injection. It offers huge emotional relief to the needle phobic and unusually pain sensitive.

Vibraject dentists say the device helps enormously with treatment as they know their patients are comfortable and the feedback they receive is overwhelmingly positive particularly from children who have nicknamed it 'the buzzy thing'. It has the potential for the next generation of children to view dental treatment very differently to their parents by eliminating the fear of injections.

www.vibraject.co.uk

\section{IMPRESSIVE IMAGES AND EASE OF USE}

At the forefront of the rapid advances in digital dentistry, Sirona have developed digital 2D and 3D X-ray systems to provide precise treatment planning.

GALILEOS and ORTHOPHOS XG 3D can offer comprehensive cone beam, panorama and cephalometric X-ray programs to suit all disciplines within dentistry.

Sirona is the only manufacturer worldwide which offers an integrated concept consisting of 3D imaging as well as computer-aided implant planning and implementation with both Galileos and the XG3D.

The ORTHOPHOS XG Family of panoramic imaging units are among the best sellers in the industry. The systems wide range of functions, excellent image quality and ease of use has impressed many users.

For further information call Sirona Dental Systems on 08450715040 or email info@ sironadental.co.uk.

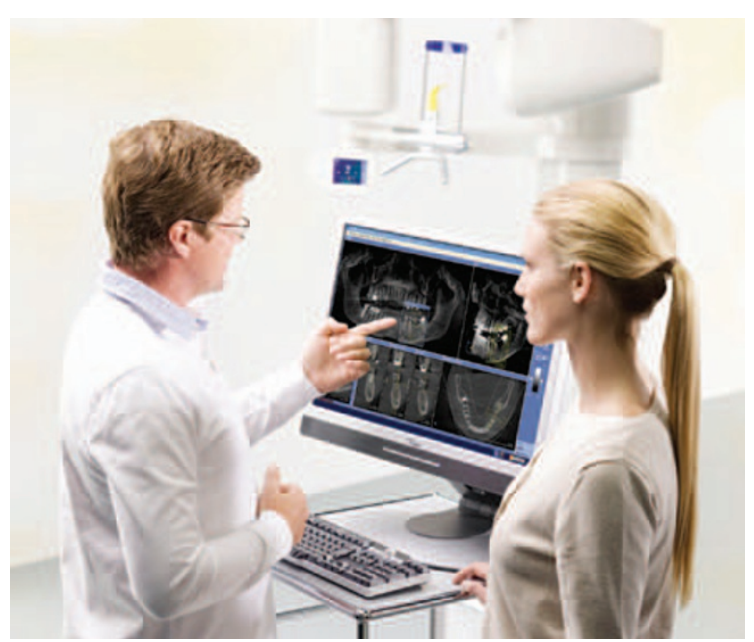

\section{DIAGNOSE IN REAL TIME WITH CONFIDENCE}

The CS 8100 from Carestream Dental offers advanced imaging technology for diagnostic excellence in one elegant and cost-effective package. An intuitive interface provides a variety of tools to help you select the right programme, position the patient, take and review the X-ray.

The desired image is acquired quickly and easily and takes mere seconds to display on screen for review. Images are clear and sharp, including: standard panoramic exam, paediatric programme, segmented panoramic, panoramic without TMJ, lateral TMJ exam, lateral TMJ exam, maxillary sinus, and exclusive $2 \mathrm{D}+$ programme

Easily adjustable, the CS 8100 can be used for patients standing or sitting, including wheelchair users, while a head support, chin rest, forehead support and handgrips ensure comfort and optimum positioning.

Ultra-compact and easy to integrate into the practice, the image quality and speed of the CS 8100 ensures real-time, accurate diagnosis with confidence.

www.carestreamdental.co.uk 